\title{
Safety and feasibility of the new 5 Fr Glidesheath Slender
}

\author{
Fuminobu Yoshimachi ${ }^{1} \cdot$ Ferdinand Kiemeneij $^{2} \cdot$ Motomaru Masutani $^{3}$. \\ Takashi Matsukage $^{4} \cdot$ Akihiko Takahashi $^{5} \cdot$ Yuji Ikari $^{1}$
}

Received: 11 March 2015 / Accepted: 23 June 2015/Published online: 4 July 2015

(C) Japanese Association of Cardiovascular Intervention and Therapeutics 2015

\begin{abstract}
The aim of this study was to evaluate the safety and feasibility of the new 5 Fr Glidesheath Slender (GSS). The transradial (TR) approach has become popular because of several advantages, such as a reduced rate of vascular access site complications. However, because the radial artery is narrow, a limitation of TR access is the potential for artery spasm or occlusion. Studies of radial artery size demonstrate that $5 \mathrm{Fr}$ sheaths are too wide for more than $10 \%$ of patients. The GSS (Terumo, Tokyo, Japan) is a new radial sheath with a thinner wall and a hydrophilic coating. It has an inner diameter that is compatible with a 5 Fr guiding catheter, while the outer diameter is similar to that of a $4 \mathrm{Fr}$ sheath. A total of 21 consecutive patients undergoing transradial angiography and/or transradial percutaneous coronary intervention with the 5 Fr GSS were included, and safety and feasibility of the device were assessed. Transradial angiography was performed in 10 patients, and transradial intervention in 11 patients. All procedures were successful, without the need for conversion to the transfemoral approach. The radial artery occlusion rate was $0 \%$, including a patient who had experienced six previous radial artery punctures. There were no cases of artery spasm,
\end{abstract}

Fuminobu Yoshimachi

yoshimachi-circ@umin.ac.jp

1 Department of Cardiology, Tokai University School of Medicine, 143 Shimo Kasuya, Isehara, Kanagawa 259-1193, Japan

2 Tergooi Hospital, Blaricum, The Netherlands

3 Department of Cardiology, Syowa Hospital, Amagasaki, Japan

4 Department of Cardiology, Tokai University Hachioji Hospital, Hachioji, Japan

5 Takahashi Hospital, Kobe, Japan hematoma, major bleeding or functional disorders. One coronary artery perforation caused by a guidewire was reported, but it was unrelated to the sheath introducer. The new 5 Fr GSS was safe and feasible for transradial angiography and transradial intervention.

Keywords Glidesheath Slender - Less invasive treatment $\cdot$ Slender percutaneous coronary intervention . Transradial angiography · Transradial coronary intervention

\section{Introduction}

Transradial angiography (TRA) and transradial coronary intervention (TRI) have become popular approaches because of several advantages, such as fewer bleeding complications [1-3]. However, use of TRA and TRI is limited for patients with a narrow radial artery, being associated with the risk of artery spasm, occlusion or pain. Indeed, the $6 \mathrm{Fr}$ sheath size is too big for some patients. The outer diameter of a $5 \mathrm{Fr}$ sheath may also be too wide because radial artery studies demonstrate that a $4 \mathrm{Fr}$ sheath is optimal for the majority of patients $[4,5]$.

Glidesheath Slender (GSS, Terumo, Japan) is compatible with a 5 Fr guiding catheter and it was designed solely for TR access. All the 5Fr Guiding Catheters, which we can use in Japan 2015, are compatible with the 5Fr GSS that was already checked in vitro. It has a thinner wall, which reduces the outer diameter by $1 \mathrm{Fr}$, while the diameter of the inner lumen is unchanged (Fig. 1). The length of the 5Fr GSS is $16 \mathrm{~cm}$. Aminian et al. reported the safety and feasibility of the 6 Fr GSS [6]. In this study, initial experience with the 5 Fr GSS (4 Fr outer diameter) was evaluated in patients undergoing TRA and TRI, focusing on safety and feasibility. 


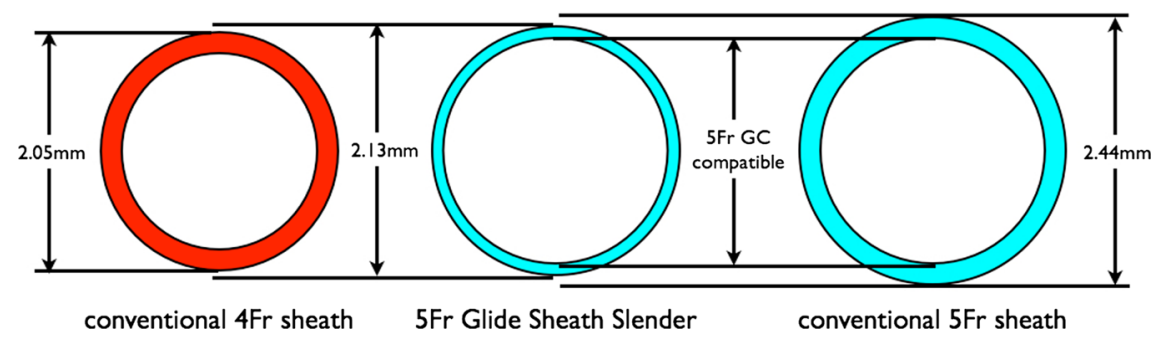

Fig. 1 The novel introducer sheath of the 5 Fr Glidesheath Slender has an inner lumen the same size as a conventional 5 Fr sheath, combined with an outer diameter similar to that of a conventional $4 \mathrm{Fr}$ sheath. The artery access size is $1 \mathrm{Fr}$ lower than that of a conventional $5 \mathrm{Fr}$ sheath

\section{Methods}

TRA or TRI using the GSS 5 Fr was performed in Tergooi Hospital, the Kingdom of the Netherlands, on two consecutive days in January 2015. Patients were enrolled prospectively, not randomized. The inclusion criterion (Fig. 2) was suspected or confirmed ischemic heart disease requiring coronary angiography or percutaneous coronary intervention (PCI) via the radial artery. Ad hoc PCI was planned for all patients, and preoperative administration of dual antiplatelet drugs was mandatory. Exclusion criteria were as follows: no indication for coronary angiography or PCI, less than 21 years old, females with the possibility of pregnancy, unable to obtain written informed consent, and patients whom the physician deemed to be unsuitable for this study.

Only procedures using the 5 Fr GSS were included. The approach was via either the right or left radial artery because the GSS is approved exclusively for radial artery access.

After local anesthesia, puncture with 21-gauge needles was performed, the guide wire was inserted, and the $5 \mathrm{Fr}$

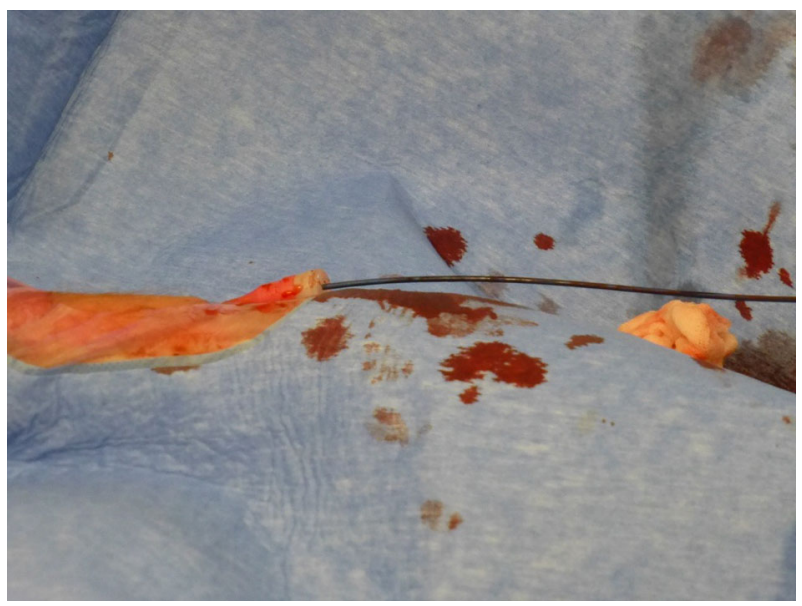

Fig. 2 5Fr GC was inserted to the 5Fr GSS via right radial approach. However, common color of $5 \mathrm{Fr}$ sheath is gray; the color of the $5 \mathrm{Fr}$ GSS is red which is for common 4Fr sheath. Because outer diameter of the 5Fr GSS is the same as that of conventional 4Fr sheath
GSS was placed inside the radial artery. All patients received $5000 \mathrm{IU}$ of heparin, irrespective of the scheduled procedure. The decision to perform ad hoc PCI after TRA was made by each physician.

Following procedure completion, the 5 Fr GSS was immediately removed, and hemostasis was performed with either TR-Band (Terumo, Japan) or a bandage and small pillow, as selected by the physician. TR-band was removed 4-5 h after confirmation of bleeding cessation, while bandages were removed after the overnight hospital stay.

\section{End points}

The primary endpoint was radial artery patency after removal of hemostasis devices, and pulsation was compared with that before the procedure. For cases with no pulsation or a low level of pulsation, radial artery patency was determined with the pulse Doppler method.

Secondary endpoints were: radial artery spasm, hematoma, major bleeding complication, functional disorder including hand ischemia due to radial artery dysfunction, pain score, and major adverse cardiac and cerebrovascular events (MACCE) related to the GSS. Radial Spasm was defined clinically as significant limitation of the catheter movement felt by the operator with severe pain in the forearm of the patient.

MACCE was defined as: all-cause mortality, cardiac death, myocardial infarction, cerebral bleeding, cerebral infarction, or acute revascularization.

Pain was assessed after sheath removal using the visual analog scale (VAS) [7], with scores ranging from 0 to $10 \mathrm{~cm}$. Large hematoma was defined as more than $5 \mathrm{~cm}$ or equal to $5 \mathrm{~cm}$ in diameter, and small hematoma as less than $5 \mathrm{~cm}$. Major bleeding was defined as fatal bleeding, and/or symptomatic bleeding in a critical area or organ, and/or bleeding accompanied by either a decline in hemoglobin levels of greater than or equal to $20 \mathrm{~g} \mathrm{~L}^{-1}$ $\left(1.24 \mathrm{mmol} \mathrm{L}^{-1}\right)$ or the need for a transfusion of greater than or equal to 2 units of whole blood or red blood cells [8]. 


\section{Results}

The clinical characteristics of patients are presented in Table 1. A total of 21 patients were included, of whom $85.7 \%$ were male. The mean age was $61.5 \pm 13.6$ (mean $\pm \mathrm{SD}$ ) years, and the mean body mass index was $26.6 \pm 3.8 \mathrm{~kg} / \mathrm{m}^{2}$. The mean number of previous attempts at radial artery access was 0.85 , and the maximum was six.

Ad hoc PCI was performed after TRA in 11 patients, while the other 10 patients underwent TRA only. The mean procedure time was $24.1 \pm 9.2 \mathrm{~min}$

The results of primary and secondary endpoints are shown in Table 2. No cases of radial artery occlusion were observed. In one patient who had undergone radial access six times previously, pulsation was very weak from the beginning of the procedure, and no change was detected after TR-Band removal; as a result, patency was assessed with the pulse Doppler method.

\section{Discussion}

This is the first report of clinical experience with the $5 \mathrm{Fr}$ GSS. No cases of radial artery occlusion or spasm, and no bleeding complications were reported in this study. VAS scores were very low, indicating that the sheath had a low propensity to cause pain. It should be noted that neither radial artery occlusion nor spasm occurred in a patient who

Table 1 Patients' characteristics $(n=21)$

\begin{tabular}{ll}
\hline Age (years) & $61.5 \pm 13.6(35-91)$ \\
Male gender $(\%)$ & $18(85.7)$ \\
Height $(\mathrm{cm})$ & $174 \pm 9.1$ \\
Weight $(\mathrm{kg})$ & $64.0 \pm 14.5$ \\
BMI $\left(\mathrm{kg} / \mathrm{m}^{2}\right)$ & $26.6 \pm 3.8$ \\
Coronary risk factor, $n(\%)$ & \\
Hypertension & $11(52.4)$ \\
Dyslipidemia & $9(42.8)$ \\
Diabetes & $6(28.6)$ \\
Insulin treated & $2(9.2)$ \\
Current smoker & $10(47.6)$ \\
Number of previous radial access, $n(\%)$ & \\
0 & $10(47.6)$ \\
1 & $8(38.1)$ \\
2 & $2(9.5)$ \\
6 & $1(7.8)$ \\
Procedure $(\%)$ & \\
Angiography & $10(47.6)$ \\
Ad hoc PCI & $11(52.4)$ \\
Procedure time (min) & $24.1 \pm 9.2$ \\
\hline
\end{tabular}

$B M I$ body mass index, $P C I$ percutaneous coronary intervention
Table 2 Results of the primary and secondary endpoints

\begin{tabular}{ll}
\hline Patency after removal of hemostasis devices $(\%)$ & \\
Patency & $21(100.0)$ \\
Radial artery occlusion & 0 \\
Spasm & 0 \\
Major and minor hematoma & 0 \\
Major bleeding & 0 \\
Functional disorder & 0 \\
Pain score & $0.57(0.0-3.0)$ \\
MACCE & 0
\end{tabular}

MACCE: all-cause mortality, cardiac death, myocardial infarction, cerebral bleeding, cerebral infarction, or acute revascularization related to the Glidesheath Slender

had undergone TR access six times previously. The outer lumen of the 5 Fr GSS is the same as that of a conventional $4 \mathrm{Fr}$ sheath; however, $5 \mathrm{Fr}$ catheters were used in this study, and the results are similar to those observed with 4 Fr conventional sheaths.

Nevertheless, it was a concern that the thinner wall of the GSS may be prone to kinking or fracture, but no such phenomenon occurred.

It is well known that the use of a sheath with a larger diameter than that of the radial artery causes injury. Reducing the sheath size from $5 \mathrm{Fr}$ to $4 \mathrm{Fr}$ is associated with safer TR access, with the rate of vascular access site complications reduced by up to 5 and $15 \%$ in Japanese males and females, respectively $[4,5]$. It is expected that a similar level of risk reduction may be achieved with the 5 Fr GSS. Another study reported that a sheath with a smaller outer diameter was more comfortable for patients, and the threshold ratio of radial artery/sheath outer diameter was 0.9 , even in cases without radial artery occlusion and painful feelings using a conventional $5 \mathrm{Fr}$ sheath [9]. For these reasons, TR access is safer and more comfortable with the 5 Fr GSS than conventional 5 Fr sheaths.

Recently, the introduction of next-generation devices has made it possible to use 5 Fr guiding catheters for routine PCI. All the available stents are compatible with a 5 Fr guiding catheter. Furthermore, the kissing balloon technique can be performed with a 5 Fr guiding catheter and 0.010 in. guide wire for bifurcation lesions [10]. PCI for chronic total occlusion is also possible [11]. Several techniques to enhance back-up support have been introduced for complex lesions, including a child's catheter [12].

Limitations of this study include the small number of cases, single ethnic origin of the Netherlands, and the use of two different hemostasis devices. Especially, Netherlander is well known for strong constitution of the body and the size of radial artery may be larger than that of Asian race. We need more studies to other race including 
Asian, who have small sized radial artery. Because this small size of the sheath seems to be useful to people having small sized radial artery to prevent radial artery occlusion or spasm. The selection of hemostasis devices by the physicians was not random. The TR-Band was mainly selected for one-day outpatient procedures because of the lack of overnight nursing staff with experience using this hemostasis device. Further studies are necessary to evaluate the 5 Fr GSS in Asian populations, which have a higher bleeding tendency.

This sheath has thin wall. In this study, there were no kinking and bending phenomenon after using angiography and intervention. But we have to know that GSS may be fragile with manipulating violently and have to be used gently to disturb breaking.

Finally, limitation of this report should be described. Using the echography and the doppler flow may clarify the impact of the 5Fr GSS on radial artery. But it was difficult to use those imaging devices, because the hospital disallowed to use it. This is the first in men of 5Fr GSS. We should make another multi center registration comparing with 5Fr GSS and conventional 5Fr sheath in order to prove the superiority of slender sheath.

\section{Conclusion}

The initial clinical experience with the new 5 Fr GSS demonstrated that it was safe and feasible for TR access. No radial artery occlusion or spasm was reported. The bleeding complication rate was also $0 \%$.

Acknowledgments We wish to thank all the members of Tergooi Hospital who provided help with this study, and all members of the Slender Club Japan for contributing to the concept of a slender sheath.

Conflict of interest All authors must disclose any affiliation with or financial involvement in any organization with a direct financial interest in the subject matter or materials discussed in the manuscript.

\section{References}

1. Feldman DN, Swaminathan RV, Kaltenbach LA, Baklanov DV, Kim LK, Wong SC, et al. Adoption of radial access and comparison of outcomes to femoral access in percutaneous coronary intervention: an updated report from the national cardiovascular data registry (2007-2012). Circulation. 2013;127:2295-306.

2. Jolly SS, Amlani S, Hamon M, Yusuf S, Mehta SR. Radial versus femoral access for coronary angiography or intervention and the impact on major bleeding and ischemic events: a systematic review and meta-analysis of randomized trials. Am Heart J. 2009;157:132-40.

3. Romagnoli E, Biondi-Zoccai G, Sciahbasi A, Politi L, Rigattieri $\mathrm{S}$, Pendenza $\mathrm{G}$, et al. Radial versus femoral randomized investigation in ST-segment elevation acute coronary syndrome: the RIFLE-STEACS (Radial versus femoral randomized investigation in ST-Elevation acute coronary syndrome) study. J Am Coll Cardiol. 2012;60:2481-9.

4. Fujii T, Masuda N, Toda E, Shima M, Tamiya S, Ito D, et al. Analysis of right radial artery for transradial catheterization by quantitative angiography-anatomical consideration of optimal radial puncture point. J Invasive Cardiol. 2010;22:372-6.

5. Saito S, Ikei H, Hosokawa G, Tanaka S. Influence of the ratio between radial artery inner diameter and sheath outer diameter on radial artery flow after transradial coronary intervention. Catheter Cardiovasc Interv. 1999;46:173-8.

6. Aminian A, Dolatabadi D, Lefebvre P, Zimmerman R, Brunner P, Michalakis $G$, et al. Initial experience with the Glidesheath Slender for transradial coronary angiography and intervention: a feasibility study with prospective radial ultrasound follow-up. Catheter Cardiovasc Interv. 2014;84:436-42.

7. Wewers ME, Lowe NK. A critical review of visual analogue scales in the measurement of clinical phenomena. Res Nurs Health. 1990;13:227-36.

8. Schulman S, Kearon C. Definition of major bleeding in clinical investigations of antihemostatic medicinal products in non-surgical patients. J Thromb Haemost. 2005;3:692-4.

9. Gwon HC, Doh JH, Choi JH, Lee SH, Hong KP, Park JE, et al. A 5Fr catheter approach reduces patient discomfort during transradial coronary intervention compared with a $6 \mathrm{Fr}$ approach: a prospective randomized study. J Interv Cardiol. 2006;19:141-7.

10. Yoshimachi F, Masutani M, Matsukage T, Saito S, Ikari Y. Kissing balloon technique within a $5 \mathrm{Fr}$ guiding catheter using 0.010 inch guidewires and 0.010 inch guidewire-compatible balloons. J Invasive Cardiol. 2007;19:519-24.

11. Matsukage T, Masutani M, Yoshimachi F, Takahashi A, Katsuki $\mathrm{T}$, Saito S, et al. A prospective multicenter registry of 0.010-inch guidewire and compatible system for chronic total occlusion: the PIKACHU registry. Catheter Cardiovasc Interv. 2010;75:1006-12.

12. Kiemeneij F, Yoshimachi F, Matsukage T, Amoroso G, Fraser D, Claessen BE, et al. Focus on maximal miniaturisation of transradial coronary access materials and techniques by the Slender Club Japan and Europe: an overview and classification. EuroIntervention. 2015;10:1178-86. 